
WITH JUVENILE IDIOPATHIC ARTHRITIS

M. Sellami, A. Fazaa, S. Miladi, K. Ouenniche, S. Kassab, S. Chekili, K. Ben Abdelghani, A. Laatar. Rheumathology, Mongi Slim Hospital - La Marsa, Tunis, Tunisia

Background: Juvenile Idiopathic Arthritis (JIA) is the most common rheumatic disease of childhood which may cause physical dysfunction and inactive sedentary lifestyle. Physical activity (PA), known to maintain optimal metabolic function and normal development could be impaired during JIA.

Objectives: The aim of this study was to assess PA in children and adolescents with JIA compared with a healthy population and to analyse associations between PA, functional ability and disease activity.

Methods: This is a cross-sectional study including patients with JIA (ILAR criteria) and age and gender-matched healthy Tunisian schoolchildren. Disease activity was evaluated by Juvenile Arthritis Disease Activity Score (JADAS) and functional ability by the Childhood Health Assessment Questionnaire (CHAQ). PA was assessed by children's physical activity questionnaire (CPAQ) for children and for adolescents (APAQ) and expressed as physical activity level (PAL). Moderate to vigorous PA (MVPA) (hours/day) and sedentary time (hours/day) were determined.

Results: A total of 55 patients (38 boys and 17 girls) with JIA and 60 healthy schoolchildren were included. No significant differences for age, height, weight, and body mass index between JIA patients and controls were observed. A quarter of children lived in poor socio-economic conditions. Subtypes of JIA were: oligoarticular $(52.7 \%)$, rheumatoid factor-negative $(16.3 \%)$ or positive $(10.9 \%)$ polyarthritis, systemic JIA (9\%), and enthesitis-related arthritis (7.2\%). The mean disease duration was 3.2 years. $^{2-6}$ Twelve patients had active disease $(21.8 \%), 25$ patients had inactive disease (45.4\%) and 18 patients were in remission $(32.7 \%)$. The mean average of $C H A Q$ was 0.98 . Seventy six percent of the JIA group spent most of the time (average of 15 hours/week) on the two lowest categories: sleeping and sitting compared with the control group (average of 8 hours/week). Only 11 children with JIA (20\%) played sport regularly with an average of 40 minutes/ week which was significantly lower compared with controls (120 minutes/week; $\mathrm{p}=0.01)$. Children with JIA spent less time in MVPA compared with the control group $(0.5 \pm 0.6$ versus $1.9 \pm 0.9$ hours/day; $p<0.01)$. Only $34.5 \%$ of JIA patients met public health recommendations to perform $\geq 1$ hour daily MVPA compared with $66.6 \%$ in the control group $(p<0.01)$. No associations were found between PA level, disease duration and disease activity. However, lower PAL in children with JIA was significantly associated with young age $(p=0.03)$, worse well-being $(p=0.01)$ and $C H A Q>1(p<0.001)$. A higher CHAQ score was associated with less time spend in MVPA $(p<0.001)$ and poor willingness to practice PA $(p=0.001)$. Sedentary time was associated with higher body mass index $(p=0.02)$.

Conclusions: In our study, children and adolescents with JIA were less physically active than the healthy peers and less active than recommended for general health. This population needs more attention in achieving normal PA.

Disclosure of Interest: None declared

DOI: 10.1136/annrheumdis-2018-eular.4750

\section{THU0590 THE ROLE OF STAT4 RS7574865 G/T AND IFR5 RS2004640 G/T GENE POLYMORPHISM IN JUVENILE IDIOPATHIC ARTHRITIS SUSCEPTIBILITY AMONG RUSSIAN PAEDIATRICPOPULATION}

M. Krylov ${ }^{1}$, E. Fedorov ${ }^{1}$, S. Salugina ${ }^{1}$, N. Konovalova ${ }^{2}$, D. Varlamov ${ }^{2} .{ }^{1}$ V.A Nasonova Research Institute of Rheumatology; ${ }^{2}$ All-Russian Research Institute of Agricultural Biotechnology,, Moscow, Russian Federation

Background: Juvenile idiopathic arthritis (JIA) is the common chronic condition in paediatric population aged under 16 y.o. JIA is an autoimmune disorder with the potential contribution from genetic factors and environmental exposures. Signal transduction and activation of transcription 4 (STAT4) gene and interferon regulatory factor 5 (IRF5) are the common set of susceptibility genes in many autoimmune disorders.

Objectives: To study STAT4 rs7574865 G/T and IFR5 rs2004640G/T gene polymorphisms impact on JIA susceptibility among Russian children.

Methods: The study included 177 subjects, 66 JIA cases and 111 healthy volunteers (the control group). Mean age of JIA patients was $11.7 \pm 4.2$ y (3-17 y), mean disease duration was $4.8 \pm 3.8$ y. All JIA patients were on the record at V. A. Nasonova Research Institute of Rheumatology, meeting ILAR-2001 criteria for JIA diagnosis. JIA group was comprised of 30 (45\%) patients with oligoarthritis (oligoJIA), 20 (30\%) patients with polyarthritis (poly-JIA) and $16(24 \%)$ patients with systemic onset arthritis (SoJIA). Oligo-JIA subgroup included 20 (67\%) HLA-B27seropositive patients (JIA-B27), and 10 (34\%) patients with anterior uveitis (JIAuveitis). The variables used for patients classification were: sex (44 girls and 22 boys); disease duration: $\leq 4$ years - $34(51 \%)$ patients and $>4$ years - $32(49 \%)$ patients; the number of joints involved: $\leq 4-29(44.0 \%)$ and $>4-37(56.0 \%)$; positive antibody titers to nuclear antigens (ANA) - 26 (39\%) patients. STAT4 and IRF5 genes polymorphism was studied using real time PCR.

Results: There was no significant difference between JIA and healthy volunteers in the incidence of various genotypes and alleles in the studied range of polymorphisms. The only significant difference was found between the rates of T STAT4 allele in oligo-JIA group and T allele in the control group (38\% and $20.4 \%$, respectively, $p=0.006)$. Similar differences with the controls in $\mathrm{T}$ allele rates were observed in JIA-B27 and JIA-uveitis subgroups $(35 \%$ and $45 \%$ vs $20.4 \%$ $p=0.044$ and $p=0.021$, respectively). Therefore, carriers of T STAT4 allele demonstrate high JIA susceptibility as compared to healthy subjects (OR $2.4395 \% \mathrm{Cl}$ (1.23-4.70), $p=0.007$ ). The risk in JIA-B27 and JIA-uveitis was (OR $2.1095 \% \mathrm{Cl}$ (0.38-4.60), $p=0.070$ and OR $3.1995 \% \mathrm{Cl}(1.09-9.06), p=0.021$, respectively) There was no specific association between studied gene polymorphisms and increased JIA susceptibility depending on sex, disease duration, number of joints involved and ANA status.

Conclusions: Obtained data confirm the specific role of STAT4 rs7574865 G/T polymorphism as the susceptibility factor to oligo-JIA associated with uveitis, HLA-B27 in Russian paediatric population.

Disclosure of Interest: None declared

DOI: 10.1136/annrheumdis-2018-eular.2424

\section{THU0591 SERUM 25 OH VITAMIN D IN PAEDIATRIC SYSTEMIC LUPUS ERYTHEMATOSUS: IN RELATION TO DISEASE ACTIVITY, DURATION AND BONE MINERAL DENSITY}

M.H. Abu-Zaid ${ }^{1}$, H.H. Abdelnabi ${ }^{2}$, A.M. El-Barbary ${ }^{1}{ }^{1}$ Rheumatology and Rehabilitation; ${ }^{2}$ Pediatric, Faculty of Medicine, Tanta University, Egypt., Tanta, Egypt

Background: Vitamin D has immunomodulatory effects and is commonly deficient in Paediatric SLE (pSLE) so associated with the disease activity and low bone density ${ }^{1,2}$

Objectives: To assess serum level of $25(\mathrm{OH})$ vitamin $\mathrm{D}$ in paediatric lupus patients and correlate it with disease activity, duration and bone density

Methods: A retrospective cohort study conducted on 90 patients with pSLE clas sified into three groups (30 patients in each) according to disease activity and duration. Group I (Initial active patients), group II (relapsing active patients) and group III (inactive patients), compared with 60 healthy children as controls (group IV). Disease and drugs duration, present steroid dose, medications' history and SLEADI for disease activity were evaluated. CBC, serum C3, C4, 24 hour urinary proteins, anti-dsDNA, Ca, P, PTH, 25(OH) D levels and lumbar spine BMD Z score were measured. Vitamin D levels were correlated with clinical, laboratory and radiological parameters.

Results: There were significant differences in mean $25(\mathrm{OH}) \mathrm{D}$ concentration between the patients' groups $(I=15.37 \pm 5.72, \|=17.70 \pm 4.88, \quad I I=26.98 \pm 6.59 \mathrm{ng}$ $\mathrm{ml}$ ) and controls $=35.90 \pm 9.66 \mathrm{ng} / \mathrm{ml}(\mathrm{p}<0.05)$, with significant difference between active groups (I, II) and inactive group III $(\mathrm{p}<0.05)$. Lumbar spine Z-score was significantly different between groups (I: $-0.39 \pm 0.81$, II: $-1.58 \pm 1.12$, III: -0.99 \pm 0.89 , IV: $0.58 \pm 0.71$ ). There were significant negative correlations between serum 25(OH) D and SLEDAI $(r-0.545, p$ 0.001), steroid dose $(r-0.561, p$ $0.001)$, anti-dsDNA ( $r-0.685, p 0.006), 24$ hr-proteinuria $(r-0.738, p 0.001)$ and PTH ( $r-0.335$, p 0.001), significant positive correlations between 25(OH) D and C3 ( $r$ 0.617, p 0.001),C4 ( $r$ 0.544, p 0.001) and serum Ca ( $\mathrm{r} 0.424, \mathrm{p} 0.001)$ with non-significant correlations between $25(\mathrm{OH}) \mathrm{D}$ and disease duration, steroids' duration, serum $P$ and $Z$ scores $(p>0.05)$.

Abstract THU0591 - Table 1. different correlations in this study

\begin{tabular}{|c|c|c|}
\hline \multirow{2}{*}{$\begin{array}{l}\text { Pearson } \\
\text { Correlation }\end{array}$} & \multicolumn{2}{|c|}{$25(\mathrm{OH})$ Vit D } \\
\hline & (r) & $\begin{array}{c}(\mathbf{P} \\
\text { value })\end{array}$ \\
\hline SLEDAI & -0.545 & $0.001^{*}$ \\
\hline Steroid dose & -0.561 & $0.001^{*}$ \\
\hline Anti-dsDNA & -0.685 & $0.006^{*}$ \\
\hline 24 hr-proteinuria & -0.738 & $0.001^{*}$ \\
\hline PTH & -0.335 & $0.001^{*}$ \\
\hline $\mathrm{C} 3$ & 0.617 & $0.001^{*}$ \\
\hline C4 & 0.544 & $0.001^{*}$ \\
\hline $\mathrm{Ca}$ & 0.424 & $0.001^{*}$ \\
\hline Disease duration & -0.215 & 0.080 \\
\hline Steroid duration & -0.089 & 0.363 \\
\hline Z-score & 0.084 & 0.431 \\
\hline $\mathbf{P}$ & 0.154 & 0.092 \\
\hline
\end{tabular}

Conclusions: Vitamin D deficiency is common in pSLE and is considered a risk factor for the disease occurrence as it is correlated significantly with the disease activity not duration. 